\title{
Kimyasal Kuplajlı Izhikevich Nöron Modelinin Lyapunov Kontrol Metodu ile Senkronizasyonu
}

\author{
Zühra Karaca $^{1 *}$, Nimet Korkmaz ${ }^{2}$,Yasemin Altuncu ${ }^{3}$, Recai Kılıç ${ }^{4}$ \\ 1* Niğde Ömer Halisdemir Üniversitesi, Mühendislik Fakültesi, Elektrik-Elektronik Mühendisliği Bölümü, Niğde, Türkiye \\ (ORCID: 0000-0002-3921-604X), zuhra.karaca@ ohu.edu.tr \\ 2 Kayseri Üniversitesi, Mühendislik, Mimarlık ve Tasarım Fakültes, Elektrik-Elektronik Mühendisliği Bölümü, Kayseri, Türkiye \\ (ORCID: 0000-0002-7419-1538), nimetkorkmaz@kayseri.edu.tr \\ ${ }^{3}$ Niğde Ömer Halisdemir Üniversitesi, Mühendislik Fakültesi, Elektrik-Elektronik Mühendisliği Bölümü, Niğde, Türkiye \\ (ORCID: 0000-0003-1517-8090), yaltuncu@ohu.edu.tr \\ ${ }^{4}$ Erciyes Üniversitesi, Mühendislik Fakültesi, Elektrik-Elektronik Mühendisliği Bölümü, Kayseri, Türkiye \\ (ORCID: 0000-0002-5069-6603), kilic@erciyes.edu.tr
}

(International Conference on Design, Research and Development (RDCONF) 2021 - 15-18 December 2021)

(DOI: $10.31590 /$ josat.1042337)

ATIF/REFERENCE: Karaca, Z., Korkmaz, N., Altuncu, Y. \& Kılıç, R. (2021). Kimyasal Kuplajlı Izhikevich Nöron Modelinin Lyapunov Kontrol Metodu ile Senkronizasyonu. Avrupa Bilim ve Teknoloji Dergisi, (32), 736-740.

\section{$\ddot{O} \mathbf{z}$}

Literatürde, elektriksel kuplajlı Izhikevich nöron modeline ait birçok çalışma olmasına rağmen kimyasal kuplajlı yapıya ait inceleme sınırlı sayıdadır. Çift yönlü kimyasal olarak kuplajlanan iki adet Izhikevich nöronunun Lyapunov kontrol yöntemiyle senkronizasyonu ilk defa bu çalışmada ele alınmıştır. Kuplajlanan nöronların kuplajlama ağırlığının etkisini gözlemlemek için standart sapma sonuçları verilmiştir. Kuplajlanan nöronlardan birine uygulanan Lyapunov kontrolörü ile, nöronların kuplajlama ağırlığından bağımsız şekilde senkron hale gelip gelmediğinin kontrolü yine standart sapma analizi vasıtası ile gözlemlenmiştir. Son olarak, Lyapunov kontrol yöntemi uygulanan sistemin, sinaptik kuplajlama ağırlık değerinin değişikliklerinden bağımsız bir şekilde senkron olarak ateşlendiği gösterilmiştir.

\section{Synchronization of the Chemically Coupled Izhikevich Neuron Model with the Lyapunov Control Method}

\begin{abstract}
Although there are many studies on the electrically coupled Izhikevich neuron model in the literature, the study of the chemically coupled structure is limited. The synchronization of bidirectional chemically coupled Izhikevich neurons with the Lyapunov control method is discussed for the first time in this study. Standard deviation results are given to observe the effect of the coupling weight of the coupled neurons. With the Lyapunov controller applied to one of the coupled neurons, the control of whether the neurons are synchronized regardless of the coupling weight was also observed by means of standard deviation analysis. Finally, it has been shown that the system with the Lyapunov control method is fired synchronously regardless of the changes in the value of the synaptic coupling weight.
\end{abstract}

Keywords: Izhikevich Neuron Model, Chemically Coupling, Synchronization, Lyapunov Control Method.

\footnotetext{
*Sorumlu Yazar: zuhra.karaca@ohu.edu.tr
} 


\section{Giriş}

Canlılarda, sinaps adı verilen özel bağlantı yollarıyla gerçekleştirilen sinir sistemi içersindeki iletişim, nöron olarak adlandırılan sinir hücreleri vasıtasıyla gerçekleşmektedir. Nöronlar arasındaki etkileşimler ve senkronizasyon, beynin nasıl çalıştığını anlamak için önemlidir. $\mathrm{Bu}$ sebeple nöronları modellemek, davranışlarını taklit etmek ve nöronların birbirlerine olan etkilerini anlamak amacıyla matematiksel olarak birçok biyolojik nöron modeli tanımlanmıştır. Literatürde; Hodgkin-Huxley(Hodgkin \& Huxley, 1952), FitzHugh-Nagumo [FitzHugh,1969], Wilson-Cowan (Wilson \& Cowan, 1972), Morris-Lecar (Morris \& Lecar, 1981), Hindmarsh-Rose (Hindmarsh et al., 1984) ve Izhikevich (Izhikevich, 2003) nöron modeli gibi adi diferansiyel denklemlerle tanımlanan nöronal ağ yapiları mevcuttur.

Nöronal aktivitelerin senkronizasyon çalışması, sinirbilimdeki en önemli konulardan biridir. Nöronlar arasındaki senkronizasyonun beynin nasıl çalıştığını anlamak için önemli olduğu düşünülmektedir. Çünkü sinir hücreleri örüntü tanıma, bilgi iletimi ve öğrenme gibi amaçlar doğrultusunda senkron davranış sergilemektedir. Canlılardaki bu bilgi aktarımı esnasında gerçekleșen senkron davranış durumu, senkronizasyon araştırmalarına ilham kaynağı olmaktadır. (Khoshkhou \& Montakhab, 2018; Cakır, 2017;Dhamala vd., 2004; La Rosa vd., 2000; Sabbagh 2000; Wang vd., 1993). Bu araştırmalara göre nöronlar arası aktivite, nöronların kolektif çalışmasının sonucu olarak ortaya çıkmaktadır (Yu ve Peng, 2006). Ayrıca, bir nöron ağında meydana gelen bozukluklar Parkinson, epilepsi, şizofreni gibi bazı hastalıklara yol açtığ 1 düşünülmektedir. Bu nedenlerden dolayı nöronların senkronizasyonu son yıllarda sıklıkla üzerinde durulan bir konu haline gelmiştir (Shi et al., 2009; Wang et al., 2006). Nöronal ağ yapılarında teorik olarak senkronizasyon durumunun sağlanması için Lyapunov kontrolü (Nguyen \& Hong, 2011), geri adımlamalı kontrol (Bin et al., 2006), geri besleme kontrolü (Zhang et al., 2007) ve kayan mod kontrolü (Che et al., 2011) gibi birçok kontrol yöntemi geliştirilmiştir.

Izhikevich nöron modeli 2003 yılında, Hodgkin-Huxley nöron modeline en yakın davranışı sergileyen ve Integrate-andFire nöron modellerinden sonra hesaplama kolaylığı ile literatüre sunulan en iyi modeldir. Referans (Izhkevich, 2003)'te, modelin parametrelerinin ayarlanarak, Izhikevich nöron modeli ile gerçek nöronların sergilediği yirmi farklı dinamik davranışın modellenebileceği gösterilmiştir. Ayrıca Izhikevich nöron modeli ile gerçekleştirilen, model parametrelerinin ayalanarak kaotik dinamiklerin elde edilmesi, merkezi desen üreteci tatbiki, nöral ă yapısı tanımlamaları gibi literaürde pek çok çalışma mevcuttur (Nobukawa \& Nishimura, 2015).

$\mathrm{Bu}$ çalışmada, ilk olarak Izhikevich nöron modeline ait Lyapunov üstelleri verilmiştir. Hesaplanan harici DC akım parametresi ' $I$ ' 'nın değeri bu çalışmanın devamında -99 olarak alınmıştır. Nöron dinamiğinin kaotik davranış sergilediği bu değerde Izhikevich nöron modeline ait zaman domeni ve faz portresi sonuçları sunulmuştur. Devamında herhangi bir kontrol metodu uygulanmayan iki adet sinir hücresi kimyasal kuplaj ile birbirine bağlanmıştır. Kuplajlanan nöronların hücre zarı potansiyellerinin kuplajlama ağırlığına bağlı değişimleri, hücre zarı potansiyelleri arasındaki standart sapma sonuçları yorumlanarak değerlendirilmiştir. Son olarak kuplajlanan nöronlardan birine Lyapunov kontrol yöntemi uygulanarak kimyasal kuplajlı nöronun senkronizasyonu ele alınmıştır. $\mathrm{Bu}$ e-ISSN: 2148-2683 kontrol yöntemi ile kuplajlanan nöronların ' $g$ ' kuplajlama parametresinden bağımsız şekilde senkron hale gelip gelmediğinin kontrolü de yine standart sapma sonuçları aracılığg ile gözlemlenmiştir. Bu gözlemlere göre kimyasal kuplajlı iki Izhikevich nöronunun senkron davranış sergilediği simülasyon sonuçlarıyla gösterilmiştir.

\section{Izhikevich Nöron Modeli}

Izhikevich nöron modeli, detaylı bir model olan HodginHuxley nöron modelinin birçok davranışını ve Integrate-and-Fire tipi nöron modellerinin hesaplama verimliliğini içermektedir. Buna ek olarak Izhikevich nöron modeli nöron tiplerinin ani yükselme ve patlama davranışlarını yeniden üretebilen bir modeldir (Izhikevich, 2003). Izhikevich nöron modeli iki adet durum denklemi ve bir çift sıfırlama durumuyla aşağıdaki gibi tanımlanmaktadır.

$$
\begin{aligned}
& \dot{v}=0.04 v^{2}+5 v+140-u+I \\
& \dot{u}=a(b v-u) \\
& v \geq 30 m V\left\{\begin{array}{c}
v \leftarrow c \\
u \leftarrow u+d
\end{array}\right.
\end{aligned}
$$

Burada v membran potansiyelini, u membran geri kazanım değişkenini, I harici giriş akımını, a ve b model parametrelerini, c sabiti, v değişkenin sıfırlama değerini ve d sabiti ise sıfırlama anında $\mathrm{u}$ değişkeninin artış miktarını ifade etmektedir. $\mathrm{Bu}$ parametrelerin farklı değerlerinde çeşitli nöron dinamikleri gözlemlenebilmektedir. $\mathrm{Bu}$ çalışmada nöronun kaotik bir davranış sergilemesi için parametreler; $a=0.2, b=2, c=-56, d=-16$, I=-99 olarak ayarlanmıştır (Nobukawa et al., 2017). Izhikevich nöron modeline ait kaotik rezonans durumları, Laypunov üstelleri vasitasıyla tanımlanabilir (Kim, 2010; Bizzari vd., 2013). Buna göre bu üstellerden en az birinin pozitif değer alması modelin kaotik davranış sergilediğini göstermektedir. $\mathrm{Bu}$ çalışmada Denklem 2'deki ayrık sistemler için Lyapunov üstelleri hesaplama yöntemi kullanılmıştır ve bu yöntemde ele alınan sistemin Jacobian matrisine ihtiyaç duyulmaktadır (Lynch, 2004).

$L=\frac{1}{n}\left(\ln \left|f_{\mu}^{l}\left(v_{1}\right)\right|+\ln \left|f_{\mu}^{l}\left(v_{2}\right)\right|+\ldots+\ln \left|f_{\mu}^{l}\left(v_{n}\right)\right|\right)$

Yukarıdaki denklemde $f_{\mu}^{l}$, ' $v^{\prime}$ 'ye bağlı değişimleri gösterirken, ' $v_{1}^{\prime},{ }^{\prime} v_{2}{ }^{\prime}, \ldots,{ }^{\prime} v_{n}{ }^{\prime}$ iterasyonları ifade etmektedir. Izhikevich nöron modeline ait Jacobian matrisi Denklem (3)'te verilmektedir.

$J=\left[\begin{array}{cc}0.08 v+5 & -1 \\ a b & -a\end{array}\right]$

Izhikevich nöron modelinin Lyapunov üstelleri $\mathrm{I}=[-110,-90]$ aralığındaki farklı ' $I$ ' harici dc akım değerleri için hesaplatılıp Şekil 1'de verilmektedir.

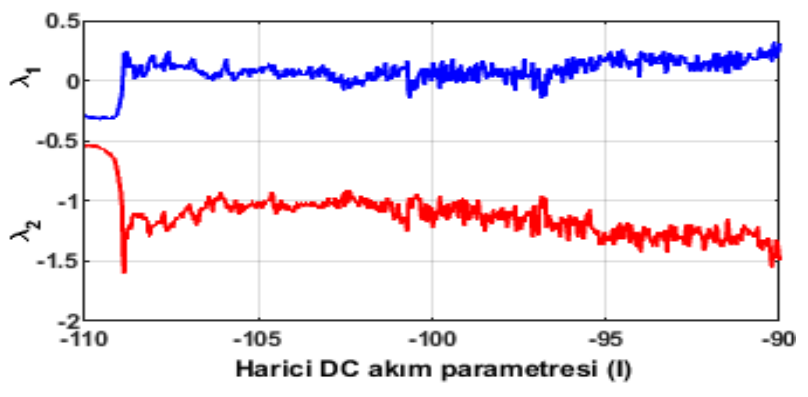

Şekil 1. $I=[-110,-90]$ aralığında farklı ' $I$ ' harici dc akım değerleri için Izhikevich nöron modeline ait Lyapunov üstelleri 737 
Şekil 1'de görüldüğü gibi I=-99 değerinde Izhikevich nöron modeli kaotik davranış sergilemektedir. $a=0.2, b=2, c=-56, d=-$ 16, I=-99 parametre değerleri için Izhikevich nöron modelinin kaotik davranışlarına ait sayısal simülasyon sonuçları Şekil 2'de sunulmaktadır.

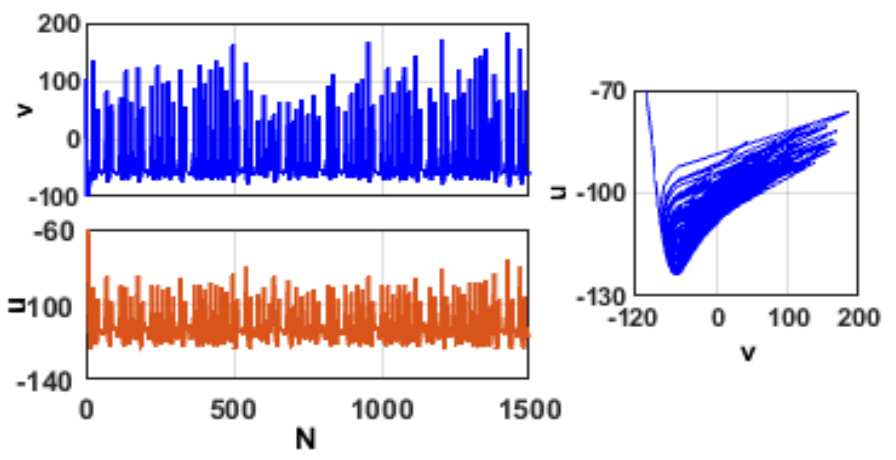

Şekil 2. Izhikevich nöron modeline ait kaotik davranış için zaman domeni ve faz portesi

\subsection{Kimyasal Kuplajlı Izhikevich Nöron Modeli}

Denklem 1 ile tanımlanan Izhikevich nöron modeli kullanılarak modellenen ve kuplajlama tipi olarak kimyasal kuplajlama kullanılan yapıya ait matematiksel modelleme Denklem 4'te verilmektedir.

$$
\begin{gathered}
\dot{v}_{i}=0.04 v_{i}^{2}+5 v_{i}+140-u_{i}+I-I_{j}^{s y n} \\
\dot{u}_{i}=a\left(b v_{i}-u_{i}\right) \\
v_{i} \geq 30 m V\left\{\begin{array}{c}
v_{i} \leftarrow c \\
u_{i} \leftarrow u_{i}+d
\end{array}\right. \\
I_{j}^{s y n}=-\sum_{1(i \neq j)} g_{j i} s_{j}\left(v_{i}-v_{s y n}\right) \\
\dot{v}_{j}=\partial\left(v_{j}\right)\left(1-s_{j}\right)-\frac{s_{j}}{\zeta} \\
\partial\left(v_{j}\right)=\frac{\partial_{0}}{1+e^{-\frac{v_{j}}{v_{s h p}}}}
\end{gathered}
$$

Burada $v_{l}$, birinci nöronun, $\mathrm{v}_{2}$ ikinci nöronun hücre zar1 potansiyellerini ifade etmektedir. Denklemde $i=1,2$ ve $j=1,2$ 'dir. ' $\mathrm{g}_{\mathrm{ji}}$ ' parametresi sinaptik ağırlık kuplajını belirtmektedir. Denklem 4'te kullanılan parametrelerin değerleri $\partial_{0}=3, \varsigma=2$, $\mathrm{V}_{\text {shp }}=5$ olarak alınmıştır. Burada $\mathrm{V}_{\text {syn }}=0$ ise sinaptik bağlantı uyarıc1 (ekshibitör) olduğunu, $\mathrm{V}_{\mathrm{syn}}<0$ ise sinaptik bağlantı engelleyici (inhibitör) olduğunu ifade etmektedir. g kuplajlama ağırlığının değerine bağlı olarak sistem senkron yada asenkron davranış sergileyebilmektedir. Bunun için kuplajlı nöronların hangi g parametresi değerlerinde senkron hangi g parametresi değerlerinde asenkron davranış sergildiğini gözlemlemek için Şekil 3'te standart sapma grafiği verilmiştir. Şekil 3'e göre $g<$ -1.1 değerinden küçük değerler için standart sapma sonuçları sıfıra yakınsamaktadır. Bu grafikte standart sapma sonuçları sıfıra yakınsıyorsa kuplajlı Izhikevich nöronları senkron ateşlenmekte, aksi değerlerde asenkron ateşlenmektedir.

Denklem 4'te verilen sisteme ait hata durum denklemleri Denklem 5 'teki gibi tanımlanabilir:

$e_{v}=v_{1}-v_{2}$
Kuplajlama parametresinin sisteme etkisini gözlemlemek için farklı $\mathrm{g}$ değerlerinde nümerik simülasyonlar yapılmıştır. $\mathrm{g}=1.5$ ve $\mathrm{g}=-1.5$ değerleri için yapılan nümerik simülasyonlar sırasıyla Şekil 4 ve Şekil 5'te verilmektedir. Buna göre kuplajlı nöronlar $\mathrm{g}=1.5$ değerinde asenkron ateşlenirken, $\mathrm{g}=-1.5$ değeri için senkron ateşlenmektedir.

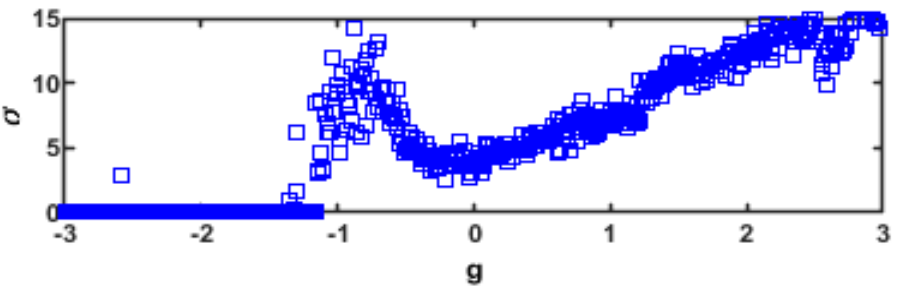

Şekil 3. İki kimyasal kuplajlı Izhikevich nöronuna ait standart sapma sonuçları
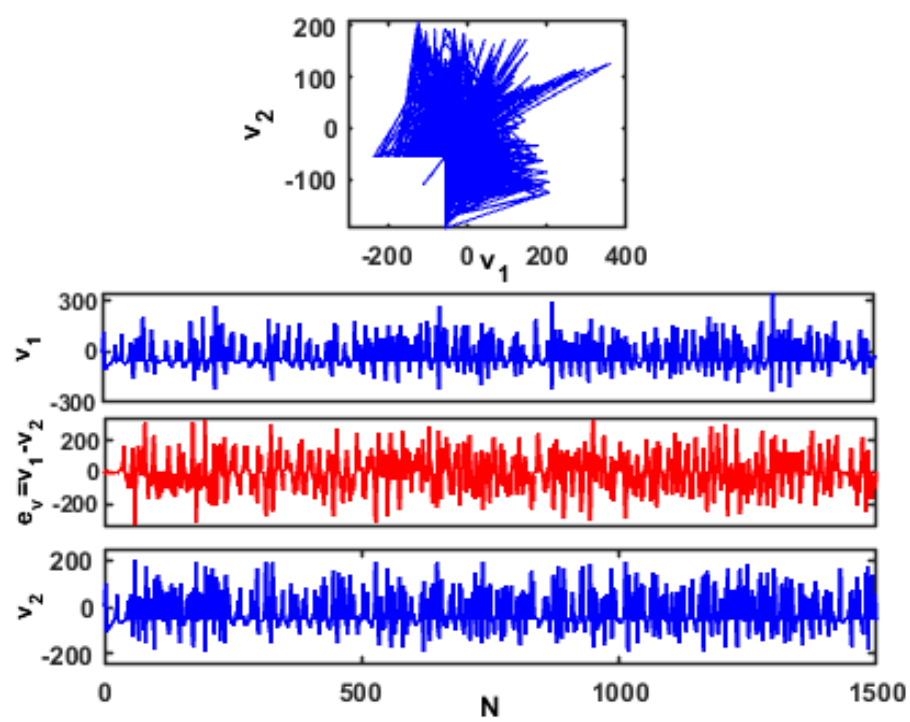

Şekil 4. İki kimyasal kuplajlı Izhikevich nöronunun sayısal simülasyon sonuçları: Kuplajlı nöronların faz portresi, zaman

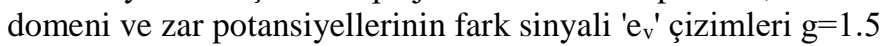
değeri için

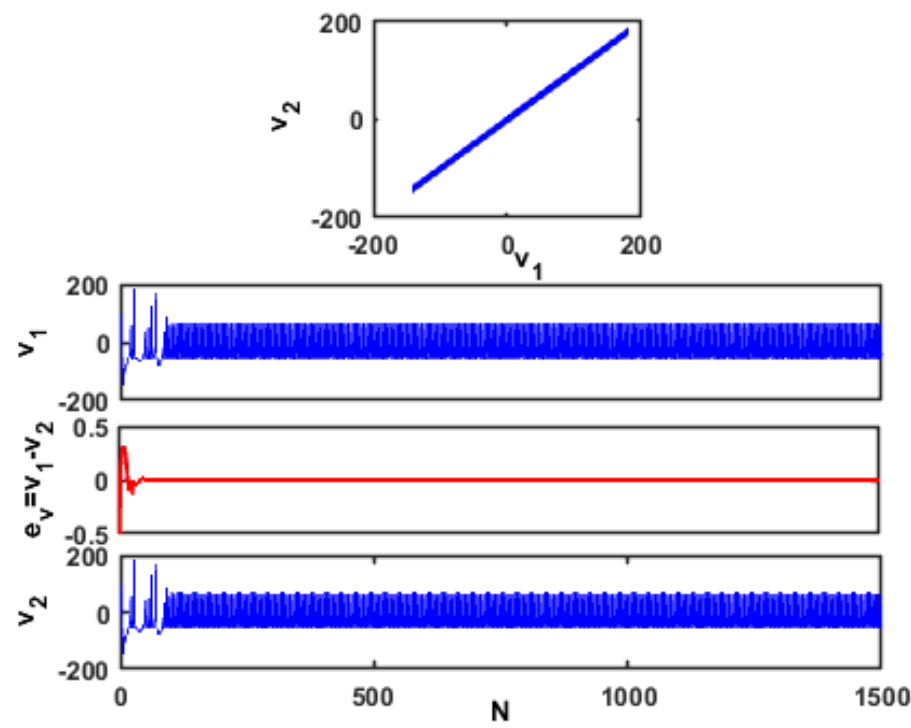

Şekil 5. İki kimyasal kuplajlı Izhikevich nöronunun sayısal simülasyon sonuçları: Kuplajlı nöronların faz portresi, zaman domeni ve zar potansiyellerinin fark sinyali ' $\mathrm{e}_{\mathrm{v}}$ ' çizimleri $\mathrm{g}=-1.5$ değeri için 


\section{Lyapunov \\ Kontrol Yöntemi \\ ile \\ Senkronizasyon}

Lyapunov kontrol yöntemi, sisteme ait bir denge noktasının kararlıllığı ya da asimptotik kararlılığ 1 ile ilgili bilgi vermektedir. Lyapunov kararlılık kriterinin diğer bir avantajı ise sisteme uygulanan kontrol parametresinin, kapalı döngü sistemi farklılaştırmamasıdır. Böylece uygulanan kontrolör hata sistemi için kapalı döngü kararlılığı garanti etmekle birlikte, senkronizasyon hatası ile ilgili de bilgi vermektedir (Kuang \& Cong, 2008; Nguyen \& Hong, 2011).

Denklem 4'te verilen kimyasal kuplajlı iki Izhikevich nöronundan oluşan sisteme ait eşitlikler aşağıdaki gibi yeniden yazılabilir.

$$
\begin{gathered}
\dot{v}_{1}=0.04 v_{1}^{2}+5 v_{1}+140-u_{1}+I-g\left(v_{1}-v_{2}\right) \\
\dot{u}_{1}=a\left(b v_{1}-u_{1}\right) \\
v_{1} \geq 30 m V \quad \Rightarrow \begin{array}{c}
v_{1} \leftarrow c \\
u_{1} \leftarrow u_{1}+d
\end{array} \\
\dot{v}_{2}=0.04 v_{2}^{2}+5 v_{2}+140-u_{2}+I-g\left(v_{2}-v_{1}\right) \\
+K \\
\dot{u}_{2}=a\left(b v_{2}-u_{2}\right) \\
v_{2} \geq 30 m V \quad \Rightarrow \quad v_{2} \leftarrow c \\
u_{2} \leftarrow u_{2}+d
\end{gathered}
$$

Denklem 6'da verilen sisteme ait hata durum denklemleri Denklem 7'deki gibi ifade edilmektedir.

$$
\begin{gathered}
\dot{e}_{v}=0.04 e_{v}\left(v_{1}+v_{2}\right)+5 e_{v}-e_{u}-K \\
-\left(I_{1}^{s y n}-I_{2}^{s y n}\right) \\
\dot{e}_{u}=a b e_{v}-a e_{u}
\end{gathered}
$$

Lyapunov kontrol yöntemine göre ilgili sistemin kararlı olup olmadığının kontrolü için, öncelikle $V=\frac{1}{2} e_{v}^{2}+\frac{1}{2} e_{u}^{2}$ şeklinde pozitif tanımlı bir enerji denklemi seçilmiştir. $\mathrm{Bu}$ denkleme ait türev ifadesi aşağıdaki gibidir.

$\dot{V}=e_{v} \dot{e}_{v}+e_{u} \dot{e}_{u}$

Denklem 8'de ilgili değişkenler yerine yazılıp, elde edilen denklemlerde sürekli pozitif değerleri veren ifadeler göz ardı edilerek, kimyasal olarak kuplajlanmış iki Izhikevich nöronuna ait Lyapunov kontrolörü ' $K$ ' nın ifadesi çekilirse, kontrolöre ait matematiksel tanımlama Denklem 9'daki gibi elde edilir:

$$
\begin{gathered}
K=0.04 e_{v}\left(v_{1}+v_{2}\right)+5 e_{v}-e_{u}-\left(I_{1}^{s y n}-I_{2}^{s y n}\right) \\
+a b e_{u}
\end{gathered}
$$

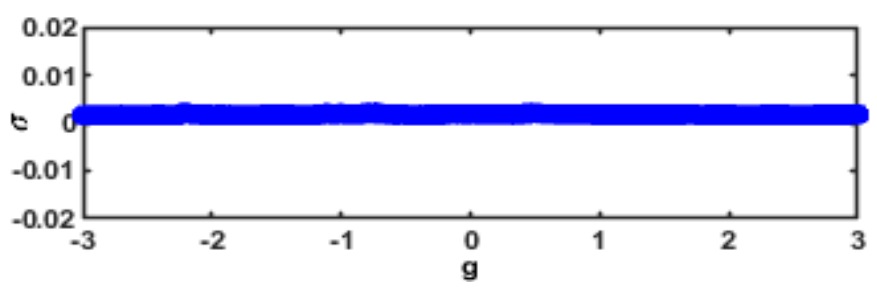

Şekil 6. Lyapunov yöntemiyle kontrol elde edilen iki kimyasal kuplajlı Izhikevich nöronuna ait standart sapma sonuçları

Denklem 9'da elde edilen ' $\mathrm{K}$ ' kontrol parametresi ikinci nörona adapte edilerek, kuplajlama ağırlığı ' $g$ ' ve diğer parametre değişimlerinden etkilenmeden, kuplajlı iki nöronun senkron ateşlenmesi elde edilmiştir. $\mathrm{Bu}$ durum Şekil 6'da verilen, Lyapunov kontrolörünün dahil edildiği kimyasal kuplajlı Izhikevich nöron modeline ait standart sapma grafiği ile Şekil 7 ve Şekil 8'de verilmektedir. Şekillerde sinaptik eşleme ağırlığı 'g'nin değeri sırasıyla g=1.5 ve g=-1.5 olarak ayarlanmıştır.
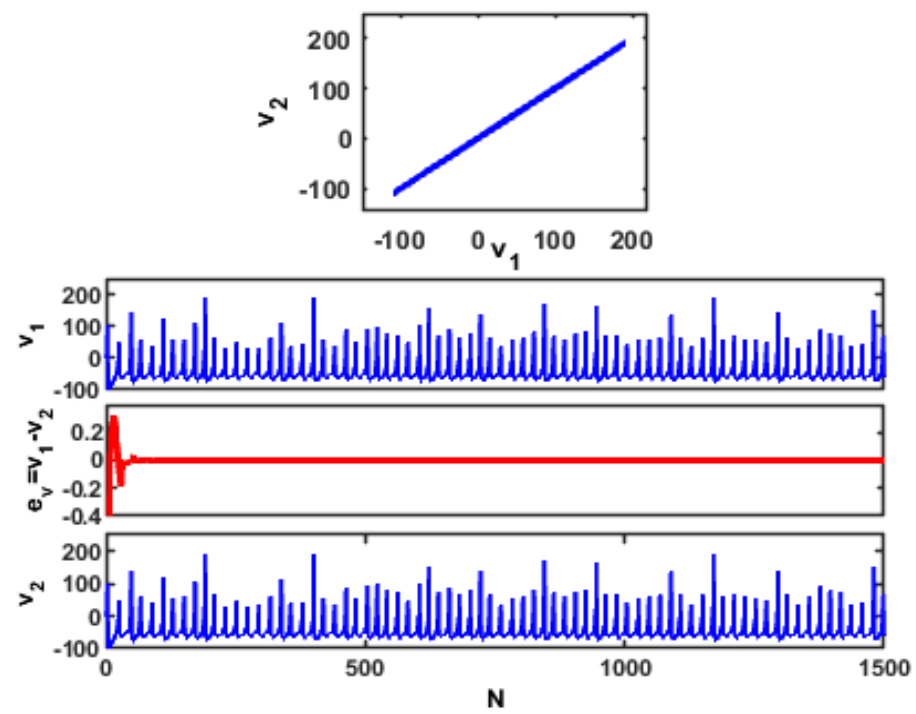

Şekil 7. Lyapunov yöntemiyle kontrol edilen iki kimyasal kuplajlı Izhikevich nöronunun sayısal simülasyon sonuçları: $\mathrm{g}=1.5$ değeri için kuplajlı nöronların faz portresi, zaman domeni ve zar potansiyellerinin fark sinyali ' $e_{\mathrm{v}}$ ' grafikleri

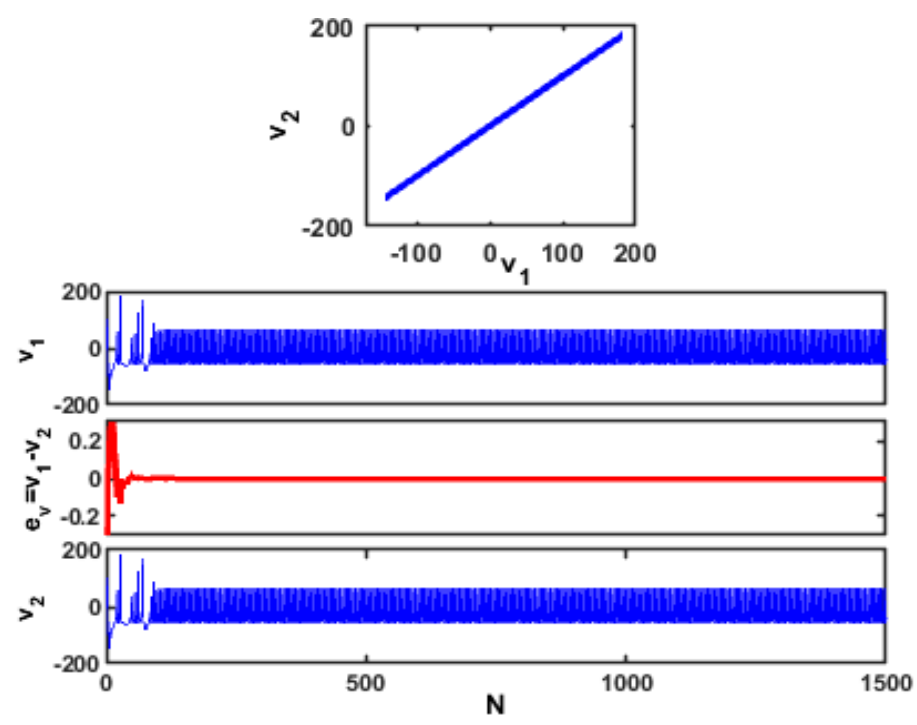

Şekil 8. Lyapunov yöntemiyle kontrol edilen iki kimyasal kuplajlı Izhikevich nöronunun sayısal simülasyon sonuçları: g=1.5 değeri için kuplajlı nöronların faz portresi, zaman domeni ve zar potansiyellerinin fark sinyali ' $\mathrm{e}_{\mathrm{v}}$ ' grafikleri

\section{Sonuç}

Bu çalışmada ilk olarak farklı harici DC akım değerlerinde Izhikevich nöron modeline ait Lyapunov üstelleri verilmiştir. Burada I=-99 değerinde Izhikevich nöron modelinin kaotik davranış sergilediği gösterilmiştir ve ' $I$ ' parametresi -99 değeri alınarak teorik simülasyonlar gerçekleştirilmiştir. Sonrasında iki Izhikevich nöron modeli birbirine kimyasal olarak kuplajlanmıştır ve modele ait standart sapma sonuçları incelenmiştir. $\mathrm{Bu}$ kontrol metodu uygulanmayan sistemin standart sapma sonucuna göre kuplajlama ağırlığı ' $g<-1.1$ değerleri için senkron davranış sergilediği, diğer değerlerde ise asenkron davranış sergilediği gözlemlenmiştir. Ardından 
Lyapunov kontrol metodu uygulanarak sistem sekron hale getirilmiştir. $\mathrm{Bu}$ kapsamda literatürde mevcut olan bu kontrol yöntemi ilk defa kimyasal kuplajlı Izhikevich nöron modeline adapte edilmiştir. Sonrasında Lyapunov kontrol metodunun sisteme dahil edildiği yapı için standart sapma sonucu incelenmiştir. $\mathrm{Bu}$ işlemin sonucunda kontrolör yapısının kullanıldı̆̆ ağırlığından bağımsız senkron ateşlendiği gözlemlenmiştir. Böylece Lyapunov kontrol yönteminin kimyasal kuplajlı iki nöron dinamiği arasında senkronizasyon durumunun elde edilmesini sağladığı gösterilmiştir.

\section{Kaynakça}

Bin, D., Jiang, W., \& Xiangyang, F. (2006). Synchronizing two coupled chaotic neurons in external electrical stimulation using backstepping control. Chaos, Solitons \& Fractals, 29(1), 182-189. https://doi.org/10.1016/j.chaos.2005.08.027

Bizzarri, F., Brambilla, A., Gajani, G. S. (2013). Lyapunov exponents computation for hybrid neurons. J. Comput. Neurosci., 35(2), 201-212. doi: 10.1007/s10827-013-04486.

Cakir, Y. (2017). Modeling of time delay-induced multiple synchronization behavior of interneuronal networks with the Izhikevich neuron model. Turk. J. Electr. Eng. Comput. Sci., 25, 2595-2605.

Che, Y., Zhang, S., Wang, J., Cui, S., Han, C., Deng, B., \& Wei, X. (2011). Synchronization of inhibitory coupled Hindmarsh-Rose neurons via adaptive sliding mode control. 2011 2nd International Conference on Intelligent Control and Information Processing, 2, 1134-1139. https://doi.org/10.1109/ICICIP.2011.6008431

Dhamala, M., Jirsa, V. K. \& Ding, M.(2004). Enhancement of neural synchrony by time delay. Phys. Rev. Lett. 92, 074104.

FitzHugh, R., Mathematical models for excitation and propagation in nerve, Schawn,H.P. (ed.) biological Engineering, McGraw-Hill, New York, 1969.

Hindmarsh, J. L., Rose, R. M., \& Huxley, A. F. (1984). A model of neuronal bursting using three coupled first order differential equations. Proceedings of the Royal Society of London. Series B. Biological Sciences, 221(1222), 87-102. https://doi.org/10.1098/rspb.1984.0024

Hodgkin, A. L., \& Huxley, A. F. (1952). A quantitative description of membrane current and its application to conduction and excitation in nerve. The Journal of Physiology, 117(4), 500-544.

Izhikevich, E. M. (2003). Simple model of spiking neurons. IEEE Transactions on Neural Networks, 14(6), 1569-1572. https://doi.org/10.1109/TNN.2003.820440

Khoshkhou, M., \& Montakhab, A. (2018). Beta-Rhythm Oscillations and Synchronization Transition in Network Models of Izhikevich Neurons: Effect of Topology and Synaptic Type. Frontiers in Computational Neuroscience, 12. https://doi.org/10.3389/fncom.2018.00059

Kim, Y. (2010).Identification of dynamical states in stimulated Izhikevich neuron models by using a 0-1 test. Journal of the Korean Physical Society, 57(6), 1363-1368. Doi: $10.3938 / \mathrm{jkps} .57 .1363$.

Kuang, S., \& Cong, S. (2008). Lyapunov control methods of closed quantum systems.Automatica,44(1),98-108. https://doi.org/10.1016/j.automatica.2007.05.013

La Rosa, M., Rabinovich, M. I., Huerta, R., Abarbanel, H. D. I. $\&$ Fortuna, L.(2000). Slow regularization through chaotic oscillation transfer in an unidirectional chain of HindmarshRose models. Phys. Lett. A 266(1), 88-93.

Lynch, S. (2004). Dynamical systems with applications using MATLAB. Boston: Birkhäuser.

Morris, C., \& Lecar, H. (1981). Voltage oscillations in the barnacle giant muscle fiber. Biophysical Journal, 35(1), 193-213. https://doi.org/10.1016/S0006-3495(81)84782-0

Nguyen, L. H., \& Hong, K.-S. (2011). Synchronization of coupled chaotic FitzHugh-Nagumo neurons via Lyapunov functions. Mathematics and Computers in Simulation, 82(4), 590-603. https://doi.org/10.1016/j.matcom.2011.10.005

Nobukawa, S., \& Nishimura, H. (2015). Stochastic resonance effects in Izhikevich neural system with spike-timing dependent plasticity. 2015 54th AnnualConference of the Society of Instrument and Control Engineers of Japan (SICE),

$270-275$ https://doi.org/10.1109/SICE.2015.7285324

Nobukawa, S., Nishimura, H., \& Yamanishi, T. (2017). Chaotic Resonance in Typical Routes to Chaos in the Izhikevich Neuron Model. Scientific Reports, 7(1), 1331. https://doi.org/10.1038/s41598-017-01511-y

Sabbagh, H. (2000). Control of chaotic solutions of the Hindmarsh-Rose equations. Chaos Soliton. Fract. 11(8), 1213-1218.

Shi, Y., Wang, J., Deng, B., \& Liu, Q. (2009). Chaotic Synchronization of Coupled Hindmarsh-Rose Neurons Using Adaptive Control. 2009 2nd International Conference on Biomedical Engineering and Informatics, 1-5. https://doi.org/10.1109/BMEI.2009.5302804

Wang, W., Perez, G. \&Cerdeira, H. A. (1993). Dynamical behavior of the firings in a coupled neuronal system. Phys. Rev. E. 47(4), 2893-2898.

Wang, Q. Y., Lu, Q. S., Chen, G. R., \& Guo, D. H. (2006). Chaos synchronization of coupled neurons with gap junctions. Physics Letters A, 356(1), 17-25. https://doi.org/10.1016/j.physleta.2006.03.017

Wilson, H. R., \& Cowan, J. D. (1972). Excitatory and Inhibitory Interactions in Localized Populations of Model Neurons. Biophysical Journal, 12(1), 1-24.

Yu, H. and Peng, J. (2006). Chaotic synchronization and control in nonlinear-coupled Hindmarsh-Rose neural systems. Chaos Soliton. Fract., 29(2), 342-348.

Zhang, T., Wang, J., Fei, X., \& Deng, B. (2007). Synchronization of coupled FitzHugh-Nagumo systems via MIMO feedback linearization control. Chaos, Solitons \& Fractals, 33(1), 194-202. https://doi.org/10.1016/j.chaos.2006.01.037 